INPP-UVA-00-03

November 20, 2000

hep-ph/0012295

\title{
Quark Orbital Angular Momentum in the Baryon
}

\author{
Xiaotong Song \\ Institute of Nuclear and Particle Physics \\ Department of Physics, University of Virginia, P.O.Box 400714 \\ Charlottesville, Virginia 22904-4714
}

\begin{abstract}
Analytical and numerical results, for the orbital and spin content carried by different quark flavors in the baryons, are given in the chiral quark model with symmetry breaking. The reduction of the quark spin, due to the spin dilution in the chiral splitting processes, is transferred into the orbital motion of quarks and antiquarks. The orbital angular momentum for each quark flavor in the proton as a function of the partition factor $\kappa$ and the chiral splitting probability $a$ is shown. The cancellation between the spin and orbital contributions in the spin sum rule and in the baryon magnetic moments is discussed.
\end{abstract}

12.39.Fe, 13.40.Em, 13.88.+e, 14.20.-c

Typeset using REVTEX 


\section{Introduction}

Since the EMC data from a deep inelastic polarized muon-polarized proton scattering experiment was published [1]], the study of spin became one of the most active areas of nuclear and particle physics. Through many experimental and theoretical investigations, spin physics has made significant progress in the past decade (see, for instance, most recent review papers [2], [3], and references therein). A basic question in the study of nucleon spin is how the total angular momentum (spin) is distributed between the intrinsic spins of the quarks and gluons and their relative orbital motion. From quantum chromodynamics (QCD) [4], the nucleon spin can be decomposed into the quark and gluon contributions

$$
\frac{1}{2}=\sum_{q}<J_{z}>_{q+\bar{q}}+<J_{z}>_{G}=\frac{1}{2} \Delta \Sigma+\sum_{q}<L_{z}>_{q+\bar{q}}+<J_{z}>_{G},
$$

where $\Delta \Sigma / 2=\sum_{q}(\Delta q+\Delta \bar{q}) / 2=\sum_{q}<s_{z}>_{q+\bar{q}}$ denotes the total contribution from the intrinsic spin of quarks and antiquarks. Note that $\Delta q \equiv q_{\uparrow}-q_{\downarrow}$, and $\Delta \bar{q} \equiv \bar{q}_{\uparrow}-\bar{q}_{\downarrow}$, and $q_{\uparrow, \downarrow}$ $\left(\bar{q}_{\uparrow, \downarrow}\right)$ are quark (antiquark) numbers with spin parallel and antiparallel to the nucleon spin, or more precisely, quark (antiquark) numbers of positive and negative helicities. $\sum_{q}<L_{z}>_{q+\bar{q}}$ denotes the total orbital angular momentum carried by quarks and antiquarks, and $\left\langle J_{z}\right\rangle_{G}$ is the gluon angular momentum. Without loss of generality, in (1) the proton has been chosen to be longitudinally polarized in the $z$ direction; it has helicity of $+1 / 2$. The total angular momentum of quarks and antiquarks $<J_{z}>_{q+\bar{q}}$ has been decomposed into the spin and orbital parts in (1).

Polarized deep-inelastic scattering (DIS) data [5] indicate that the intrinsic quark spins only contribute about one third of the nucleon spin or even less. The smallness of $\frac{1}{2} \Delta \Sigma$ implies that the missing part should be contributed either by the quark orbital motion or the gluon angular momentum. There is no direct data on $\Delta G$ except for a preliminary restriction on $\Delta G(x) / G(x)$ given by experiment E581/704 [6], and another indirect result $\Delta G \simeq 0.5-$ 1.5 at $Q^{2} \simeq 10 \mathrm{GeV}^{2}$ from the analysis of $Q^{2}$ dependence of $g_{1}\left(x, Q^{2}\right)$ [7]. Several experiments for measuring $\Delta G$ [8] have been suggested. For the orbital motion, it has been shown that $\left.<J_{z}\right\rangle_{q+\bar{q}}$ can be measured in the deep virtual Compton scattering (DVCS) [9], and one may then obtain the quark orbital angular momentum from the difference $\left\langle J_{z}>_{q+\bar{q}}-\left\langle s_{z}\right\rangle_{q+\bar{q}}\right.$. Since the nucleon is a complex composite object of quarks and gluons, the configuration of these elementary constituents is determined by their mutual interactions governed by the underlying theory of strong interactions - QCD. Study of different components in (1) and how they share the nucleon spin will give invaluable information on nonperturbative structure of the nucleon.

Historically, when the quark model [10] was invented in 1960's, all three quarks in the nucleon were assumed to be in S-states, so that $\left\langle L_{z}\right\rangle_{q}=0$ and the nucleon spin arises entirely from the quark spin. On the other hand, in the simple parton model [11], all quarks, antiquarks and gluons are moving in the same direction, i.e. parallel to the proton momentum, there is no transverse momentum for the partons and thus $\left\langle L_{z}>_{q+\bar{q}}=0\right.$ and $<L_{z}>_{G}=0$. This picture cannot be $Q^{2}$ independent due to QCD evolution. In leading-log approximation, $\Delta \Sigma$ is $Q^{2}$ independent, but the gluon helicity $\Delta G$ increases with $Q^{2}$. This increase should be compensated by the decrease of the orbital angular momentum carried by partons (see for instance Ref. [12 and later analysis [13). Recently, the leading-log 
evolution of $\left\langle L_{z}>_{q+\bar{q}}\right.$ and $\left\langle L_{z}>_{G}\right.$, and an interesting asymptotic partition rule were obtained in |14. The 'initial' value of the orbital angular momenta at the renormalization scale $\mu^{2}$ are determined by nonperturbative dynamics of the nucleon as a QCD bound state. Lattice QCD provides us with a nonperturbative tool to calculate the physical quantities of hadrons and leads to many interesting results [15]. Meantime, many QCD inspired nucleon models have been developed to explain existing data and yield good physical insight into the nucleon. For instance, in the bag model [16, [17], $\sum_{q}<s_{z}>_{q} \simeq 0.38$, and $\sum_{q}<L_{z}>_{q} \simeq 0.12$, while in the Skyrme model [18], $\Delta G=\Delta \Sigma=0$, and $\left\langle L_{z}\right\rangle=1 / 2$, which implies that the nucleon spin arises only from the orbital motion.

Phenomenologically, long before the EMC experimental data [1] were published, using the Bjorken sum rule and low energy hyperon $\beta$-decay data, Sehgal [19] showed that nearly $40 \%$ of the nucleon spin arises from the orbital motion of quarks, the remaining $60 \%$ is attributed to the spin of quarks and antiquarks. Most recently Casu and Sehgal [20] show that to fit the baryon magnetic moments and polarized DIS data, a large collective orbital angular momentum $\left\langle L_{z}>\right.$, which contributes almost $80 \%$ of nucleon spin, is needed. Hence the question remains of how much of the nucleon spin is coming from the quark orbital motion.

Recently, the chiral quark model was successfully used to explain [21] both the smallness of $\Delta \Sigma$ and a flavor-asymmetry of the light-quark sea of the nucleon $(\bar{d}-\bar{u} \neq 0)$. The model was improved by introducing $\mathrm{U}(1)$-breaking [22] and the kaonic suppression [23]. Several modified descriptions with both $\mathrm{SU}(3)$ and $\mathrm{U}(1)$-breaking were developed in 24 28. The quark orbital motion in the chiral quark model was discussed in 29] (SU(3) symmetry) and [30] (SU(3) breaking) respectively. In [29], authors found that due to significant cancellation between the contributions from the sea spin polarization and the orbital angular momentum, the naive quark model can yield a good account of the baryon magnetic moments. In [30], an unified formalism for describing both spin and orbital angular momentum was suggested. However, an 'equal sharing' assumption was used in [30] and needs to be improved. In this paper, the partition factor $\kappa$ [see section III (a)] is no longer restricted to be $1 / 3$ and can be any value in the range of $[0,1 / 2]$. In section II, the basic assumptions and formalism are briefly reviewed. In section III, we give the formalism for calculating both spin and orbital angular momentum carried by quarks and antiquarks in the nucleon. Generalization to the octet and decuplet baryons is given in section IV. Numerical results are also given. The magnetic moments are discussed in section V. Different scenarios of the proton spin decomposition and a brief summary are given in section VI.

\section{Chiral Quark Model}

The nonperturbative dynamics of quarks and gluons inside the nucleon is closely related to the QCD vacuum structure. At the energies lower than the nucleon mass $(\sim 1 \mathrm{GeV})$, the QCD vacuum has nonperturbative condensations of quarks [31] and gluons [32]. The nonzero vacuum expectation values imply that the $S U(3) \times S U(3)$ chiral symmetry is broken down to $S U(3)$ flavor symmetry. This phenomenon is manifested in the existence of eight Goldstone bosons $(\pi, K, \eta)$ and the existence of dynamical mass in the current quark propagator. Hence the bare massless (current) quarks become massive (constituent) quarks, which make a good basis to represent the QCD Hamiltonian at low energies. In the chiral quark model, the important degrees of freedom are quarks and Goldstone bosons, and the dominate interaction is the coupling among quarks and Goldstone bosons, while the gluon effect is expected to 
be rather small $\left(<J_{z}>_{G} \neq 0\right.$ case will be discussed briefly in the later sections). In this description, the structure of the nucleon is determined by the valence quark structure of the nucleon and all possible quark-Goldstone boson fluctuations. The sea of quarks and antiquarks in this model is generated from the Goldstone bosons and not from gluons.

Following the notations used in [27] and [30], the effective Lagrangian is

$$
L_{I}=g_{8} \bar{q}\left(\begin{array}{ccc}
G_{u}^{0} & \pi^{+} & \sqrt{\epsilon} K^{+} \\
\pi^{-} & G_{d}^{0} & \sqrt{\epsilon} K^{0} \\
\sqrt{\epsilon} K^{-} & \sqrt{\epsilon} \bar{K}^{0} & G_{s}^{0}
\end{array}\right) q,
$$

where $G_{u(d)}^{0}$ and $G_{s}^{0}$ are defined as

$$
G_{u(d)}^{0}=+(-) \frac{\pi^{0}}{\sqrt{2}}+\sqrt{\epsilon_{\eta}} \frac{\eta^{0}}{\sqrt{6}}+\zeta^{\prime} \frac{\eta^{\prime 0}}{\sqrt{3}}, \quad G_{s}^{0}=-\sqrt{\epsilon_{\eta}} \frac{\eta^{0}}{\sqrt{6}}+\zeta^{\prime} \frac{\eta^{\prime 0}}{\sqrt{3}},
$$

and the breaking effects are explicitly included. We use $a \equiv\left|g_{8}\right|^{2}$ to denote the transition probability of chiral fluctuation or splitting $u(d) \rightarrow d(u)+\pi^{+(-)}$, then $\epsilon a$ denotes the probability of $u(d) \rightarrow s+K^{-(0)}$. Similar definitions are used for $\epsilon_{\eta} a$ and $\zeta^{\prime 2} a$. If the breaking parameter is dominated by the mass suppression effect, one reasonably expects $0 \leq \zeta^{\prime 2} a<\epsilon_{\eta} a \simeq \epsilon a \leq a$, then we have $0 \leq \zeta^{\prime 2} \leq 1,0 \leq \epsilon_{\eta} \leq 1$, and $0 \leq \epsilon \leq 1$. We note that the parameter $\zeta^{\prime}$ must be negative which is required from the experimental data (see discussion in [24]). In our numerical calculation, we use the approximation $\epsilon_{\eta} \simeq \epsilon$ given in [27], so that only three parameters remain. However, our analytical results and conclusions do not depend on this approximation. We also note that in our formalism, only the integrated quark spin and flavor content are discussed.

The basic assumptions of the chiral quark model are: (i) The quark flavor, spin and orbital content of the nucleon are determined by its valence quark structure and all possible chiral fluctuations, and probabilities of these fluctuations depend on the interaction Lagrangian (2a-b). (ii) The coupling between the quark and Goldstone boson (GB) is rather weak, one can treat the fluctuation $q \rightarrow q^{\prime}+\mathrm{GB}$ as a small perturbation $(a \simeq 0.10-0.15)$ and contributions from the higher order fluctuations can be neglected. (iii) The quark spin-flip interaction dominates the splitting process $q \rightarrow q^{\prime}+$ GB. This can be related to the picture given by the instanton model [33] and the spin-nonflip interaction is suppressed.

Based upon these assumptions, the quark flips its spin and changes (or maintains) its flavor by emitting a charged (or neutral) Goldstone boson. The light quark sea asymmetry $\bar{u}<\bar{d}$ is attributed to the existing flavor asymmetry of the valence quark numbers (two valence $u$-quarks and one valence $d$-quark) in the proton. On the other hand, the quark spin reduction is due to the spin dilution in the chiral splitting processes. Furthermore, the quark spin component changes one unit of angular momentum, $\left(s_{z}\right)_{f}-\left(s_{z}\right)_{i}=+1$ or -1 , due to spin-flip in the fluctuation with GB emission. The angular momentum conservation requires the same amount change of the orbital angular momentum but with opposite sign, i.e. $\left(L_{z}\right)_{f}-\left(L_{z}\right)_{i}=-1$ or +1 . This induced orbital motion is distributed among the quarks and antiquarks, and compensates the spin reduction in the chiral splitting. This is the starting point to calculate the orbital angular momentum carried by quarks and antiquarks in the chiral quark model.

For spin-up or spin-down valence $u, d$, and $s$ quarks, up to the first order fluctuation, 
the allowed chiral processes are

$$
\begin{array}{r}
u_{\uparrow,(\downarrow)} \rightarrow d_{\downarrow,(\uparrow)}+\pi^{+}, \quad u_{\uparrow,(\downarrow)} \rightarrow s_{\downarrow,(\uparrow)}+K^{+}, \quad u_{\uparrow,(\downarrow)} \rightarrow u_{\downarrow,(\uparrow)}+G_{u}^{0}, \quad u_{\uparrow,(\downarrow)} \rightarrow u_{\uparrow,(\downarrow)} . \\
d_{\uparrow,(\downarrow)} \rightarrow u_{\downarrow,(\uparrow)}+\pi^{-}, \quad d_{\uparrow,(\downarrow)} \rightarrow s_{\downarrow,(\uparrow)}+K^{0}, \quad d_{\uparrow,(\downarrow)} \rightarrow d_{\downarrow,(\uparrow)}+G_{d}^{0}, \quad d_{\uparrow,(\downarrow)} \rightarrow d_{\uparrow,(\downarrow)}, \\
s_{\uparrow,(\downarrow)} \rightarrow u_{\downarrow,(\uparrow)}+K^{-}, \quad s_{\uparrow,(\downarrow)} \rightarrow d_{\downarrow,(\uparrow)}+\bar{K}^{0}, \quad s_{\uparrow,(\downarrow)} \rightarrow s_{\downarrow,(\uparrow)}+G_{s}^{0}, \quad s_{\uparrow,(\downarrow)} \rightarrow s_{\uparrow,(\downarrow)} .
\end{array}
$$

We note that the quark spin flips in the chiral splitting processes $q_{\uparrow,(\downarrow)} \rightarrow q_{\downarrow,(\uparrow)}+\mathrm{GB}$, i.e. the first three processes in each of $(3 \mathrm{a}),(3 \mathrm{~b})$, and $(3 \mathrm{c})$, but not for the last one. In the zeroth approximation, the $\mathrm{SU}(3) \otimes \mathrm{SU}(2)$ proton wave function gives

$$
n_{p}^{(0)}\left(u_{\uparrow}\right)=\frac{5}{3}, \quad n_{p}^{(0)}\left(u_{\downarrow}\right)=\frac{1}{3}, \quad n_{p}^{(0)}\left(d_{\uparrow}\right)=\frac{1}{3}, \quad n_{p}^{(0)}\left(d_{\downarrow}\right)=\frac{2}{3} .
$$

the spin-up and spin-down quark (or antiquark) content, up to first order fluctuation, can be written as

$$
n_{p}\left(q_{\uparrow, \downarrow}^{\prime}, \text { or } \bar{q}_{\uparrow, \downarrow}^{\prime}\right)=\sum_{q=u, d} \sum_{h=\uparrow, \downarrow} n_{p}^{(0)}\left(q_{h}\right) P_{q_{h}}\left(q_{\uparrow, \downarrow}^{\prime}, \text { or } \bar{q}_{\uparrow, \downarrow}^{\prime}\right)
$$

where $P_{q_{\uparrow, \downarrow}}\left(q_{\uparrow, \downarrow}^{\prime}\right)$ and $P_{q_{\uparrow, \downarrow}}\left(\bar{q}_{\uparrow, \downarrow}^{\prime}\right)$ are probabilities of finding a quark $q_{\uparrow, \downarrow}^{\prime}$ or an antiquark $\bar{q}_{\uparrow, \downarrow}^{\prime}$ arise from all chiral fluctuations of a valence quark $q_{\uparrow, \downarrow} \cdot P_{q_{\uparrow, \downarrow}}\left(q_{\uparrow, \downarrow}^{\prime}\right)$ and $P_{q_{\uparrow, \downarrow}}\left(\bar{q}_{\uparrow, \downarrow}^{\prime}\right)$ can be obtained from the effective Lagrangian (2) and listed in Table I, where only $P_{q_{\uparrow}}\left(q_{\uparrow, \downarrow}^{\prime}\right)$ and $P_{q_{\uparrow}}\left(\bar{q}_{\uparrow, \downarrow}^{\prime}\right)$ are shown. Those arise from $q_{\downarrow}$ can be obtained by using the relations, $P_{q_{\downarrow}}\left(q_{\uparrow, \downarrow}^{\prime}\right)=$ $P_{q_{\uparrow}}\left(q_{\downarrow, \uparrow}^{\prime}\right)$ and $P_{q_{\downarrow}}\left(\bar{q}_{\uparrow, \downarrow}^{\prime}\right)=P_{q_{\uparrow}}\left(\bar{q}_{\downarrow, \uparrow}^{\prime}\right)$. The notations given in Table I are defined as

$$
A \equiv 1-\zeta^{\prime}+\frac{1-\sqrt{\epsilon}_{\eta}}{2}, \quad B \equiv \zeta^{\prime}-\sqrt{\epsilon}_{\eta}, \quad C \equiv \zeta^{\prime}+2 \sqrt{\epsilon}_{\eta}
$$

and

$$
f \equiv \frac{1}{2}+\frac{\epsilon_{\eta}}{6}+\frac{\zeta^{\prime 2}}{3}, \quad f_{s} \equiv \frac{2 \epsilon_{\eta}}{3}+\frac{\zeta^{\prime 2}}{3}
$$

The special combinations $A, B$, and $C$ stem from the quark and antiquark contents in the octet and singlet neutral bosons $G_{u(d)}^{0}$ and $G_{s}^{0}$ [see $\left.(2 \mathrm{~b})\right]$ appeared in the effective chiral Lagrangian (2a), while $f$ and $f_{s}$ stand for the probabilities of the chiral splittings $u_{\uparrow}\left(d_{\uparrow}\right) \rightarrow$ $u_{\downarrow}\left(d_{\downarrow}\right)+G_{u(d)}^{0}$ and $s_{\uparrow} \rightarrow s_{\downarrow}+G_{s}^{0}$ respectively. Although there is no valence $s$ quark in the proton and neutron, there are one or two valence $s$ quarks in $\Sigma$ or $\Xi$, or other strange decuplet baryons, and even three valence $s$ quarks in the $\Omega^{-}$. Hence for the purpose of later use we also give the probabilities arise from a valence $s$-quark splitting. In general, the suppression effects may be different for different baryons, hence the probabilities $P_{q_{\uparrow, \downarrow}}\left(q_{\uparrow, \downarrow}^{\prime}\right)$ and $P_{q_{\uparrow, \downarrow}}\left(\bar{q}_{\uparrow, \downarrow}^{\prime}\right)$ may vary with the baryons. But we will assume that they are universal for all baryons.

Using (4), (5) and probabilities listed in Table I, the spin-up and spin-down quark and antiquark content, and the spin average and spin weighted quark and antiquark content in the proton were obtained in [24,27] and are now collected in Table II. For the purpose of later discussion, we write down the formula for the spin-weighted quark content

$$
\left(\Delta q^{\prime}\right)^{B}=\sum_{q}\left[n_{B}^{(0)}\left(q_{\uparrow}\right)-n_{B}^{(0)}\left(q_{\downarrow}\right)\right]\left[P_{q_{\uparrow}}\left(q_{\uparrow}^{\prime}\right)-P_{q_{\uparrow}}\left(q_{\downarrow}^{\prime}\right)\right] .
$$


Note that the spin-weighted antiquark content is zero

$$
\left(\Delta \bar{q}^{\prime}\right)^{B}=0
$$

Hence one has $(\Delta q)_{\text {sea }} \neq \Delta \bar{q}$ in the chiral quark model. This is different from those models, in which the sea quark and antiquark with the same flavor are created as a pair from the gluon (see discussion on Eq.(3.12) in [23]). The quark spin contents in the proton are

$$
\Delta u^{p}=\frac{4}{5} \Delta_{3}-a, \quad \Delta d^{p}=-\frac{1}{5} \Delta_{3}-a, \quad \Delta s^{p}=-\epsilon a,
$$

where $\Delta_{3} \equiv \Delta u^{p}-\Delta d^{p}=\frac{5}{3}[1-a(\epsilon+2 f)]$. The total quark spin content in the proton is

$$
\frac{1}{2} \Delta \Sigma^{p}=\frac{1}{2}\left(\Delta u^{p}+\Delta d^{p}+\Delta s^{p}\right)=\frac{1}{2}-(1+\epsilon+f) a \equiv \frac{1}{2}-\xi_{1} a,
$$

where the notation $\xi_{1} \equiv 1+\epsilon+f$ is introduced. The total loss of quark spin $(1+\epsilon+f) a$ appeared in Eq.(7d) is due to the fact that there are three splitting processes with quark spinflip [see the first three processes in (3a) and (3b)], probabilities of these spin-flip splittings are $a, \epsilon a$, and $f a$ respectively.

\section{Quark Orbital Motion}

(a) Quark orbital angular momentum in the nucleon

The quark orbital angular momentum can be discussed in a similar way. For instance, for a spin-up valence $u$-quark, only first three processes in (3a), i.e. quark fluctuations with GB emission, can induce a change of the orbital angular momentum. The last process in (3a), $u_{\uparrow} \rightarrow u_{\uparrow}$ means no chiral fluctuation and it makes no contribution to the orbital motion and will be disregarded. The orbital angular momentum produced in the splitting $q_{\uparrow} \rightarrow q_{\downarrow}^{\prime}+\mathrm{GB}$ is shared by the recoil quark $\left(q^{\prime}\right)$ and the Goldstone boson (GB). If we define the fraction of the orbital angular momentum shared by the recoil quark is $1-2 \kappa$, then the orbital angular momentum shared by the (GB) is $2 \kappa$ which, we assume, equally shared by the quark and antiquark in the Goldstone boson. We call $\kappa$ the partition factor, which can take any value in the range $[0,1 / 2]$. For $\kappa=1 / 3$, the three particles - the quark and antiquark in the (GB) and the recoil quark - equally share the induced orbital angular momentum. This is the 'equal sharing' case discussed in [30].

We define $<L_{z}>_{q^{\prime} / q_{\uparrow}}\left(<L_{z}>_{\bar{q}^{\prime} / q_{\uparrow}}\right)$ as the orbital angular momentum, carried by the quark $q^{\prime}$ (antiquark $\bar{q}^{\prime}$ ), arises from all fluctuations of a valence spin-up quark except for no-splitting case. Considering the quark spin component changes one unit of angular momentum in each splitting and using Table I, all $\left\langle L_{z}>_{q^{\prime} / q_{\uparrow}}\right.$ and $\left\langle L_{z}>_{\bar{q}^{\prime} / q_{\uparrow}}\right.$ for $q=u, d, s$ are obtained and listed in Table III.

The orbital angular momentum produced from a spin-down valence quark splitting is the same as that from a spin-up valence quark splitting, but with opposite sign

$$
<L_{z}>_{q^{\prime} / q_{\downarrow}}=-<L_{z}>_{q^{\prime} / q_{\uparrow}}, \quad<L_{z}>_{\bar{q}^{\prime} / q_{\downarrow}}=-<L_{z}>_{\bar{q}^{\prime} / q_{\uparrow}},
$$

where both $q_{\uparrow}^{\prime}$ and $q_{\downarrow}^{\prime}$ are included in $\left\langle L_{z}>_{q^{\prime} / q_{\uparrow, \downarrow}}\right.$ (the same is true for $\left\langle L_{z}>_{\bar{q}^{\prime} / q_{\uparrow, \downarrow}}\right.$ ).

Having obtained the orbital angular momenta carried by different quark flavors produced from the spin-up and spin-down valence quark fluctuations, it is easy to write down the total 
orbital angular momentum carried by a specific quark flavor, for instance, $u$-quark, in the proton

$$
<L_{z}>_{u}^{p}=\sum_{q=u, d}\left[n_{p}^{(0)}\left(q_{\uparrow}\right)-n_{p}^{(0)}\left(q_{\downarrow}\right)\right]<L_{z}>_{u / q_{\uparrow}},
$$

where $\sum$ summed over valence $u$ - and $d$-quarks in the proton. $n_{p}^{(0)}\left(q_{\uparrow}\right)$ and $n_{p}^{(0)}\left(q_{\downarrow}\right)$ are given in (4). Similarly, one obtains the $\left\langle L_{z}>_{d}^{p},<L_{z}>_{s}^{p}\right.$, and those for antiquarks.

The analytical results are

$$
\begin{gathered}
\sum_{q}<L_{z}>_{q}^{p}=(1-\kappa) \xi_{1} a, \\
\sum_{\bar{q}}<L_{z}>_{\bar{q}}^{p}=\kappa \xi_{1} a, \\
\sum_{q}<L_{z}>_{q+\bar{q}}^{p} \equiv \sum_{q}<L_{z}>_{q}^{p}+\sum_{\bar{q}}<L_{z}>_{\bar{q}}^{p}=\xi_{1} a .
\end{gathered}
$$

Several remarks are in order:

- The amount $\xi_{1} a$ in (10c) is exactly the same as the total spin reduction in $(7 \mathrm{~d})$. The sum of $(10 \mathrm{c})$ and $(7 \mathrm{~d})$ gives

$$
\sum_{q}<J_{z}>_{q+\bar{q}}^{p}=\sum_{q}\left[<s_{z}>_{q+\bar{q}}^{p}+<L_{z}>_{q+\bar{q}}^{p}\right]=\frac{1}{2} .
$$

Therefore, in the chiral fluctuations, the missing part of the quark spin is transferred into the orbital motion of quarks and antiquarks. The amount of quark spin reduction $(1+\epsilon+f) a$ in $(7 \mathrm{~d})$ is canceled by the equal amount increase of the quark orbital angular momentum in (10c), and the total angular momentum of nucleon is unchanged.

- The orbital angular momentum of quarks or antiquarks may depend on the partition factor $\kappa$ [see(10a-b)], but the total orbital angular momentum (10c) is independent of $\kappa$. This is because the total transfer of the angular momentum from 'spin' into 'orbital' should be entirely determined by the chiral splitting not the partition factor.

- From (10b), the orbital angular momentum carried by antiquarks $\sum_{\bar{q}}<L_{z}>_{\bar{q}}$ increases with $\kappa$. This is because a larger $\kappa$ means a larger portion of induced orbital angular momentum is transfered to the Goldstone boson, and thus to the antiquark. On the contrary, $\sum_{q}<L_{z}>_{q}$ decreases with $\kappa$ [see (10a)].

- Although the orbital angular momentum carried by quarks ( antiquarks) $\sum_{q}<L_{z}>_{q}^{p}$ $\left(\sum_{\bar{q}}<L_{z}>_{\bar{q}}^{p}\right)$ depends on both $\kappa$ and the chiral parameters, the ratio of $\sum_{q}^{q}<L_{z}>_{q}^{p}$ and $\sum_{q}<L_{z}>_{\bar{q}}^{p}$ depends only on the partition factor $\kappa$

$$
\sum_{q}<L_{z}>_{q}^{p} / \sum_{q}<L_{z}>_{\bar{q}}^{p}=(1-\kappa) / \kappa
$$

For $\kappa=1 / 3$ (equal sharing), this ratio is $2: 1$. This is originated from the mechanism of the chiral fluctuation, there are two quarks and one antiquark in the final state. 
- Contrary to (11a), the ratio of quark spin and orbital content is

$$
\sum_{q}<s_{z}>_{q+\bar{q}}^{p} / \sum_{q}<L_{z}>_{q+\bar{q}}^{p}=\frac{1}{2 \xi_{1} a}-1,
$$

which is independent of $\kappa$, but depends on the chiral parameters.

The discussion can be easily extended to other baryons. Note that different baryons have different valence quark structure and thus different $n_{B}^{(0)}\left(q_{\uparrow}\right)$ and $n_{B}^{(0)}\left(q_{\downarrow}\right)$. For the neutron, explicit calculations show that $<L_{z}>_{u, \bar{u}}^{n}=<L_{z}>_{d, \bar{d}}^{p},\left\langle L_{z}>_{d, \bar{d}}^{n}=<L_{z}>_{u, \bar{u}}^{p}\right.$, and $<L_{z}>_{s, \bar{s}}^{n}=<L_{z}>_{s, \bar{s}}^{p}$. Using these relations, one can obtain the orbital angular momenta carried by quarks and antiquarks in the neutron. We have similar relations for $\Delta q$ from the isospin symmetry, hence results (7d), (10a-10d), (11a), (11b), and related conclusions hold for the neutron as well. Extension to other octet and decuplet baryons will be given in section IV.

(b) Numerical results

To determine model parameters, we use similar approach given in [27], where the chiral quark model with only three parameters gave a good description to most existing spin and flavor observables. The chiral parameters $a, \epsilon \simeq \epsilon_{\eta}$, and $\zeta^{\prime}$ are determined by three inputs, $\Delta u-\Delta d=1.26$ [34], $\Delta u+\Delta d-2 \Delta s=0.60$ [34], and $\bar{d}-\bar{u}=0.143$ [35]. The good agreement between the model prediction and spin-flavor data can be seen from Table IV. The three-parameter set is: $a=0.145, \epsilon=0.46$, and $\zeta^{\prime 2}=0.10$. It gives

$$
\xi_{1} \equiv 1+\epsilon+f=2.07 .
$$

Numerical results of the spin and orbital angular momentum shared by each quark flavor are listed in Table V. Several comments are in order:

- From (10c) and (12), one obtains

$$
\sum_{q}<L_{z}>_{q+\bar{q}}^{p} \simeq 0.30,
$$

i.e., nearly $60 \%$ of the proton spin is coming from the orbital motion of quarks and antiquarks, and $40 \%$ is contributed by the quark and antiquark spins. Comparison of our result with other models is given in Table VI and Fig. 1.

- Using the parameter set given above, we have $\epsilon a \simeq \epsilon_{\eta} a \simeq 0.067$ and $\zeta^{\prime 2} a \simeq 0.015$. It implies that probabilities of chiral splittings $q \rightarrow q^{\prime} \pi, q \rightarrow q^{\prime} K(\eta)$, and $q \rightarrow q^{\prime} \eta^{\prime}$ are $14-15 \%, 6-7 \%$, and $1-2 \%$ respectively.

- We plot the orbital angular momenta carried by quarks and antiquarks in the proton as functions of $\kappa$ in Fig.2. It shows that $\left\langle L_{z}>_{s}^{p}=<L_{z}>_{s}^{p}\right.$ at $\kappa=1 / 3$. This is because there is no valence $s$ quark in the nucleon. Hence there is no recoil $s$ quark in the chiral splitting, all $s$ and $\bar{s}$ can only come from the GB emission.

- Comparing our choice of parameters with two extreme cases,

$$
\begin{aligned}
& \xi_{1} \equiv 1+\epsilon+f=3.0, \quad \text { for } \mathrm{U}(3)-\operatorname{symmetry}\left(\epsilon=\epsilon_{\eta}=\zeta^{\prime 2}=1\right) \\
& \xi_{1} \equiv 1+\epsilon+f=1.5, \quad \text { for extreme breaking }\left(\epsilon=\epsilon_{\eta}=\zeta^{\prime 2}=0\right)
\end{aligned}
$$

we see that the value $\xi_{1}$ given in (12) is just between those given in (14a) and (14b). 
- As indicated in Eq.(10d), the total quark spin reduction is canceled by the equal increase of the orbital angular momentum. However, the exact cancellation does not apply to each quark flavor. Using the superscript (0) denotes the quantity in NQM, one has $\Delta u^{p(0)}=4 / 3, \Delta d^{p(0)}=-1 / 3$, and $\Delta s^{p(0)}=0$. From Table V, taking $\kappa=1 / 3$, we have in the chiral quark model

$$
\begin{gathered}
\frac{1}{2} \Delta u^{p}+<L_{z}>_{u+\bar{u}}^{p}=0.558<\frac{1}{2} \Delta u^{p(0)}=0.667, \\
\frac{1}{2} \Delta d^{p}+<L_{z}>_{d+\bar{d}}^{p}=-0.080>\frac{1}{2} \Delta d^{(0) p}=-0.167, \\
\frac{1}{2} \Delta s^{p}+<L_{z}>_{s+\bar{s}}^{p}=0.022>\frac{1}{2} \Delta s^{(0) p}=0 .
\end{gathered}
$$

We see that for the $u$-flavor, the orbital contribution is not big enough to compensate the quark spin reduction, but for both $d$-flavor and $s$-flavor the orbital contribution is too big and the spin reduction is over-compensated. However, taking the sum, one has

$$
\sum_{q}\left[\frac{1}{2} \Delta q^{p}+<L_{z}>_{q+\bar{q}}^{p}\right]=\frac{1}{2}=\sum_{q} \frac{1}{2} \Delta q^{p(0)} .
$$

- If we define $\Delta q_{\text {sea }} \equiv \Delta q-\Delta q_{v a l}$ and identify $\Delta q_{v a l}=\Delta q^{(0)}$ which are determined from the NQM, then

$$
\begin{aligned}
& \frac{1}{2} \Delta u_{\text {sea }}^{p}+<L_{z}>_{u+\bar{u}}^{p}=-0.109, \\
& \frac{1}{2} \Delta d_{\text {sea }}^{p}+<L_{z}>_{d+\bar{d}}^{p}=0.087 \\
& \frac{1}{2} \Delta s_{\text {sea }}^{p}+<L_{z}>_{s+\bar{s}}^{p}=0.022 .
\end{aligned}
$$

They are nonzero. Hence the exact cancellation does not occur for each quark flavor, though it does for the sum of all quark flavors, i.e.

$$
\sum_{q}\left[\frac{1}{2} \Delta q_{s e a}^{p}+<L_{z}>_{q+\bar{q}}^{p}\right]=0 .
$$

\section{Extension to other Baryons}

\section{(a) Spin content in octet baryons}

We take $\Sigma^{+}$(uus) as an example, other octet baryons can be discussed in a similar manner. The valence quark structure of $\Sigma^{+}$is the same as the proton with the replacement $d \rightarrow s$. Hence one has

$$
n_{\Sigma^{+}}^{(0)}\left(u_{\uparrow}\right)=\frac{5}{3}, \quad n_{\Sigma^{+}}^{(0)}\left(u_{\downarrow}\right)=\frac{1}{3}, \quad n_{\Sigma^{+}}^{(0)}\left(s_{\uparrow}\right)=\frac{1}{3}, \quad n_{\Sigma^{+}}^{(0)}\left(s_{\downarrow}\right)=\frac{2}{3} .
$$

Using (5) (with replacement, $p \rightarrow \Sigma^{+}$and $\sum_{q=u, d} \rightarrow \sum_{q=u, s}$ ), (16), and Table I, we can obtain $\Delta u^{\Sigma^{+}}, \Delta d^{\Sigma^{+}}$, and $\Delta s^{\Sigma^{+}}$. Similarly, we can obtain results for $\Sigma^{0}, \Lambda^{0}$, and $\Xi^{0}$. Those for $\Sigma^{-}$, and $\Xi^{-}$, can be obtained by using the isospin symmetry relations. The analytical expressions of all $\Delta q^{B}$ are listed in the upper half of Table VII. A few remarks are in order. 
- The total spin content of quarks and antiquarks in the octet baryons can be written as

$$
\sum_{q}<s_{z}>_{q+\bar{q}}^{B}=\frac{1}{2}-\frac{a}{3}\left(c_{1} \xi_{1}+c_{2} \xi_{2}\right)
$$

where $\xi_{2} \equiv 2 \epsilon+f_{s}$ [definition of $f_{s}$ see Eq.(6b)] and $\left(c_{1}, c_{2}\right)=(3,0),(4,-1),(0,3)$, and $(-1,4)$ for $\mathrm{B}=\mathrm{N}, \Sigma, \Lambda$, and $\Xi$ respectively. One can see that the spin reductions for all members in the same isospin multiplet are the same, but they are different for different isospin multiplets, except for the SU(3)-symmetry limit $\left(\xi_{1}=\xi_{2}=2+f\right)$ and $\mathrm{U}(3)$-symmetry limit $\left(\xi_{1}=\xi_{2}=3\right.$, in this case, $\left.\sum_{q}<s_{z}>_{q+\bar{q}}^{N, \Sigma, \Xi}=\frac{1}{2}-3 a\right)$.

- Using parameters $\xi_{1} \simeq 2.07$, and $\xi_{2} \simeq 1.27$, we plot the quark and antiquark spin content in different octet baryons as functions of the parameter $a$ in Fig.3. Taking $a \simeq 0.145$, one obtains

$$
\sum_{q}<s_{z}>_{q+\bar{q}}^{B} \simeq 0.20,0.16,0.32,0.35 \quad(B=N, \Sigma, \Lambda, \Xi) .
$$

- The spin of the $\Lambda$ : In the naive $\mathrm{SU}(3)$ symmetric quark model, all the spin of the $\Lambda$ comes from the spin of the $s$ quark

$$
\Delta u^{\Lambda}=\Delta d^{\Lambda}=0, \quad \Delta s^{\Lambda}=1, \quad \sum_{q} \Delta q^{\Lambda}=1 .
$$

From Table VIII, the chiral quark model predicts

$$
\Delta u^{\Lambda}=\Delta d^{\Lambda}=-0.07, \quad \Delta s^{\Lambda}=0.77, \quad \sum_{q} \Delta q^{\Lambda}=0.64 .
$$

This can compare with the result, $\Delta u^{\Lambda}=\Delta d^{\Lambda}=-0.23, \Delta s^{\Lambda}=0.58$, and $\sum_{q} \Delta q^{\Lambda}=$ 0.12 given in [36]. Our result indicates that about $64 \%$ of the $\Lambda$ spin is contributed by the spin of quarks, which include $s$ quark (positive, $+77 \%$ ) and $u, d$ quarks (negative, total $-13 \%)$. The remaining part $(36 \%)$ comes from the orbital angular momentum of quarks and antiquarks in our model and possibly from the gluon contributions in a modified chiral quark model [37] with the gluon mixing [38].

\section{(b) Orbital angular momentum in octet baryons}

Similar to the nucleon case, the orbital angular momentum carried by quarks and antiquarks in other octet baryons can be calculated. The analytical results for different isospin multiplets are listed in the lower half of Table VII. The total orbital angular momentum carried by all quarks and and antiquarks in the baryon $B$ can be written as

$$
\sum_{q}<L_{z}>_{q+\bar{q}}^{B}=\frac{a}{3}\left(c_{1} \xi_{1}+c_{2} \xi_{2}\right)
$$

where $c_{1}$ and $c_{2}$ are the same as defined in Eq. (17). The sum of spin (17) and orbital angular momentum (19) gives

$$
\sum_{q}\left[<s_{z}>_{q+\bar{q}}^{B}+<L_{z}>_{q+\bar{q}}^{B}\right]=\frac{1}{2}, \quad(B=N, \Sigma, \Lambda, \Xi)
$$


Once again, the loss of the quark spin is compensated by the gain of the orbital motion of quarks and antiquarks. Results and conclusions obtained in section III for the nucleon hold for other octet baryons as well. Numerical results of the spin and orbital angular momentum for each quark flavor in the baryon octet are listed in Table VIII. We make some remarks here.

- From (18) and (19), one can see that nearly $68 \%(36 \%, 30 \%)$ of $\Sigma^{+}\left(\Lambda, \Xi^{0}\right)$ spin is coming from the orbital motion of quarks and antiquarks, and $32 \%(64 \%, 70 \%)$ is contributed by the quark and antiquark spins. This result does not depend on the partition factor $\kappa$.

- Using relations $\left\langle L_{z}>_{u, d}^{\Sigma^{-}, \Xi^{-}}=<L_{z}>_{d, u}^{\Sigma^{+}, \Xi^{0}}\right.$ and $\left\langle L_{z}>_{s}^{\Sigma^{-}, \Xi^{-}}=<L_{z}>_{s}^{\Sigma^{+}, \Xi^{0}}\right.$ from the isospin symmetry, one can obtain orbital angular momenta in $\Sigma^{-}$and $\Xi^{-}$. Similar to the nucleon case, we have $\left\langle L_{z}>_{d}^{\Sigma^{+}, \Xi^{0}}=<L_{z}>_{\bar{d}}^{\Sigma^{+}, \Xi^{0}}\right.$ at $\kappa=1 / 3$. This is because there is no valence $d$ quark in $\Sigma^{+}$and $\Xi^{0}$, hence all $d$ and $\bar{d}$ must come from the GB.

- Similar to the nucleon case [see $\Delta_{3}$ expression below Eq.(7c)], we have a general formula for the octet baryons

$$
\Delta u^{B}-\Delta d^{B}=c_{B}[1-(\epsilon+2 f)],
$$

where $c_{B}=5 / 3,4 / 3$, and $-1 / 3$ for $B=p, \Sigma^{+}$, and $\Xi^{0}$ respectively.

- It is easy to check from the analytic results given in Table VII that the following identity holds for the $u$-quark spin

$$
\Delta u^{p}-\Delta u^{n}+\Delta u^{\Sigma^{-}}-\Delta u^{\Sigma^{+}}+\Delta u^{\Xi^{0}}-\Delta u^{\Xi^{-}}=0
$$

and the same identity holds for the $d$-quark and $s$-quark spins. One can also show by explicit calculation that the orbital angular momentums $\left\langle L_{z}\right\rangle_{u}^{B}$ in the octet baryons satisfy similar identity

$$
<L_{z}>_{u}^{p}-<L_{z}>_{u}^{n}+<L_{z}>_{u}^{\Sigma^{-}}-<L_{z}>_{u}^{\Sigma^{+}}+<L_{z}>_{u}^{\Xi^{0}}-<L_{z}>_{u}^{\Xi^{-}}=0
$$

Similar relations hold for $\left\langle L_{z}>_{d}^{B}\right.$, $<L_{z}>_{s}^{B}$, and $<L_{z}>_{\bar{q}}^{B}$. Combining (22a) and (22b), one obtains a sum rule for the magnetic moments [see Eq.(25) below] in the baryon octet

$$
\mu_{p}-\mu_{n}+\mu_{\Sigma^{-}}-\mu_{\Sigma^{+}}+\mu_{\Xi^{0}}-\mu_{\Xi^{-}}=0 .
$$

This sum rule was discussed in 28] without the orbital contributions. Our result shows that the sum rule (22c) holds in the symmetry breaking chiral quark model even the orbital contributions are included. Quark spin content, without the orbital angular momentum, in octet baryons were discussed in [28,39].

\section{(c) Decuplet baryons}

The above discussion can be extended to the baryon decuplet. The analytical results are listed in Table IX. The explicit calculation shows that

$$
(\Delta u)^{\Delta^{-}}=(\Delta d)^{\Delta^{++}},(\Delta u)^{\Delta^{0}}=(\Delta d)^{\Delta^{+}},(\Delta u)^{\Sigma^{*-}}=(\Delta d)^{\Sigma^{*+}},(\Delta u)^{\Xi^{*-}}=(\Delta d)^{\Xi^{* 0}} .
$$

They are due to the isospin symmetry of the decuplet baryon wave functions. Therefore, in Table IX, we only list results for $\Delta^{++}, \Delta^{+}, \Sigma^{*+}, \Sigma^{* 0}, \Xi^{* 0}$, and $\Omega^{-}$. A few remarks are in order. 
- From Table IX, one obtains

$$
\sum_{q}<s_{z}>_{q+\bar{q}}^{B^{*}}=\frac{3}{2}-a\left[3 \xi_{1}+S\left(\xi_{1}-\xi_{2}\right)\right],
$$

where the $S$ is the strangeness quantum number of the decuplet baryon $B^{*}$. Eq.(23a) leads to an equal spacing rule for the total quark spin in the baryon decuplet, and the spacing is

$$
\sum_{q}<s_{z}>_{q+\bar{q}}^{\Omega-\Xi}=\sum_{q}<s_{z}>_{q+\bar{q}}^{\Xi-\Sigma}=\sum_{q}<s_{z}>_{q+\bar{q}}^{\Sigma-\Delta}=a\left(\xi_{1}-\xi_{2}\right) .
$$

The equal spacing rule (23b) was also discussed in [28] without the orbital contributions.

- From (23a), for the strangeless $\Delta$ multiplet, $S=0$, one obtains

$$
\sum_{q}<s_{z}>_{q+\bar{q}}^{\Delta}=3\left[\frac{1}{2}-\frac{a}{3}\left(3 \xi_{1}\right)\right]=3 \sum_{q}<s_{z}>_{q+\bar{q}}^{N},
$$

i.e. total spin content of $\Delta$ baryon is three times that of the nucleon, this is a reasonable result.

- From Table IX, the total orbital angular momentum carried by quarks and antiquarks can be written as

$$
\sum_{q}<L_{z}>_{q+\bar{q}}^{B^{*}}=a\left[3 \xi_{1}+S\left(\xi_{1}-\xi_{2}\right)\right] .
$$

Similar to the total quark spin case, Eq.(24a) leads to an equal spacing rule for the orbital angular momentum. The spacing is

$$
\sum_{q}<L_{z}>_{q+\bar{q}}^{\Omega-\Xi}=\sum_{q}<L_{z}>_{q+\bar{q}}^{\Xi-\Sigma}=\sum_{q}<L_{z}>_{q+\bar{q}}^{\Sigma-\Delta}=-a\left(\xi_{1}-\xi_{2}\right),
$$

which is the same as (23b) but with opposite sign. The sum of spin (23a) and orbital angular momentum (24a) gives

$$
\sum_{q}\left[<L_{z}>_{q+\bar{q}}^{B^{*}}+<s_{z}>_{q+\bar{q}}^{B^{*}}\right]=\frac{3}{2} .
$$

Once again, the spin reduction is compensated by the increase of orbital angular momentum and keep the total angular momentum of the baryon (now is 3/2 for the decuplet!) unchanged.

\section{Baryon Magnetic Moments}

The baryon magnetic moment depends on both spin and orbital motions of quarks and antiquarks. In the chiral quark model all antiquark sea polarizations are zero, the baryon magnetic moment can be written as

$$
\mu_{B\left(B^{*}\right)}=\sum_{q} \mu_{q}\left[(\Delta q)^{B\left(B^{*}\right)}+<L_{z}>_{q}^{B\left(B^{*}\right)}-<L_{z}>_{\bar{q}}^{B\left(B^{*}\right)}\right] \equiv \sum_{q} \mu_{q} C_{q}^{B},
$$


where $B\left(B^{*}\right)$ denote the octet (decuplet) baryons and $\mu_{q}$ s are the magnetic moments of quarks. We have assumed that the magnetic moment of the baryon is the sum of spin and orbital magnetic moments of individual charged particles (quarks or antiquarks). The assumption of the additivity is commonly believed to be a good approximation for a loosely bound system, which is the basic description for the baryon in the chiral quark model. In addition, the baryon may contain other neutral particles, such as gluons (e.g. see discussion in [40]). Although the gluon does not make any contribution to the magnetic moment, the existence of intrinsic gluon would change the valence quark structure of the baryon due to the spin and color couplings between the gluon and quarks. In this paper, we assume that the gluon effect is small and will be neglected in our calculation. The quark spin and orbital angular momentum in a modified chiral quark model with the gluon mixing has been discuused in [37].

To calculate the baryon magnetic moment, we need to know the spin content $\Delta q$ (note that $\Delta \bar{q}=0$ in the chiral quark model) and the difference between the orbital angular momentum carried by the quark $q$ and that carried by corresponding antiquark $\bar{q}$. This difference is denoted by $<L_{z}>_{q-\bar{q}}^{B} \equiv<L_{z}>_{q}^{B}-<L_{z}>_{\bar{q}}^{B}$. For example, one has for the $u$-quark

$$
<L_{z}>_{u-\bar{u}}^{B}=\sum_{q}\left[n_{B}^{(0)}\left(q_{\uparrow}\right)-n_{B}^{(0)}\left(q_{\downarrow}\right)\right]\left[<L_{z}>_{u / q_{\uparrow}}-<L_{z}>_{\bar{u} / q_{\uparrow}}\right] .
$$

Similar equations hold for the $d$-quark, s-quark, and corresponding antiquarks.

(a) Octet baryons

Substituting (7a) and (26) into (25), one can obtain the analytic expressions of the magnetic moments for the octet baryons. It is easy to verify that they satisfy the following sum rules

$$
\begin{gathered}
(4.70) \quad \mu_{p}-\mu_{n}=\mu_{\Sigma^{+}}-\mu_{\Sigma^{-}}-\left(\mu_{\Xi^{0}}-\mu_{\Xi^{-}}\right) \\
\text {(3.66) } \quad-6 \mu_{\Lambda}=-2\left(\mu_{p}+\mu_{n}+\mu_{\Xi^{0}}+\mu_{\Xi^{-}}\right)+\left(\mu_{\Sigma^{+}}+\mu_{\Sigma^{-}}\right)
\end{gathered}
$$

where the numerical values shown in parentheses are taken from the data 34. Relations (27a) [or (22c)] and (27b) were first given by Franklin in [43]. Linear sum rules (27a), (27b), and nonlinear sum rule (27c) are not new and violated at about $10-15 \%$ level. They have been discussed in many works, for instance [44 46]. However, the new relation (27d) is rather well satisfied. Our result shows that if the $S U(3) \otimes S U(2)$ valence quark structure is used, the chiral fluctuation cannot change these sum rules even orbital contributions are included. We note that the sum rules (27a-27c) also hold for more general case [46].

Numerical results of applying Eq.(25) to the magnetic moments of the baryon octet are listed in Table X. We find that if we choose parameters $\mu_{u}, \mu_{d}$ and $\mu_{s}$ by fitting the measured values of $\mu_{p}, \mu_{n}$ and $\mu_{\Lambda}$ as is also done in the simple SU(6) quark model (NQM) [34], then results for all different $\kappa$ values are completely identical with the NQM results. Three remarks are in order.

- According to the fitting procedure, for a given set of $\mu_{u, d, s}^{(0)}$ in the NQM, there is a corresponding set of $\mu_{u, d, s}$ which gives the same values of $\mu_{p}, \mu_{n}$ and $\mu_{\Lambda}$ in the chiral quark model. 
- Seven magnetic moments of the baryon octet satisfy four sum rules, Eqs.(27a-27d), which hold for both the chiral quark model and the NQM and appear to be reasonably well satisfied. Hence, as soon as three baryon magnetic moments are taken to be the same in both cases, the remaining four should agree as well. Hence, effects of the chiral splitting with or without the orbital contribution can be absorbed into the quark magnetic moments. It implies that for describing the baryon magnetic moments, the NQM is as good as the chiral quark model. This is similar to the conclusion given in [29]. Our result shows that the magnetic moment is presumably not a suitable observable for revealing the quark orbital angular momentum in the nucleon, unless the quark magnetic moments are given.

- We have shown in section III, the total amount of quark spin reduction is canceled by the equal amount increase of the quark orbital angular momentum $\left\langle L_{z}\right\rangle_{q+\bar{q}}$, but this exact cancellation does not occur for each quark flavor. To see whether similar cancellation occurs in the baryon magnetic moments, we turn into Eq.(25). The relevant quantity is $C_{q}^{B} \equiv \Delta q^{B}+<L_{z}>_{q-\bar{q}}^{B}$ defined in Eq.(25). Using superscripts (0), (1), and (2) to denote the quantity in NQM, $\chi \mathrm{QM}$ with, and without $\left\langle L_{z}\right\rangle_{q-\bar{q}}$ respectively, we have

$$
\begin{gathered}
C_{u}^{(0) p}=4 / 3, \quad C_{d}^{(0) p}=-1 / 3, \quad C_{s}^{(0) p}=0, \quad\left(\mathrm{NQM}, \quad<L_{z}>_{q}=<L_{z}>_{\bar{q}}=0\right), \\
C_{u}^{(1) p}=0.996, C_{d}^{(1) p}=-0.403, C_{s}^{(1) p}=-0.067,\left(\chi \mathrm{QM}, \text { with }<L_{z}>_{q-\bar{q}}\right) \\
C_{u}^{(2) p}=0.863, C_{d}^{(2) p}=-0.397, C_{s}^{(2) p}=-0.067,\left(\chi \mathrm{QM}, \text { without }<L_{z}>_{q-\bar{q}}\right) .
\end{gathered}
$$

The orbital contribution does move $C_{u}^{(2) p}$ up to $C_{u}^{(1) p}$, but it is still far below $4 / 3$. For the $d$-quark flavor, the orbital contribution moves $C_{d}^{(2) p}$ down and $C_{d}^{(1) p}$ is even more negative than $-1 / 3$. Comparing (28a-28c) with (15a-15c), one can see that in the proton magnetic moment, there is a partial cancellation between the contributions from the quark spin reduction and the quark orbital angular momentum for the $u$ flavor $\left(<L_{z}>_{u-\bar{u}}^{p}=0.133\right)$, but no cancellation for the $s$-flavor $\left(<L_{z}>_{s-\bar{s}}^{p}=0\right)$. For the $d$-flavor, the orbital contribution even enhances the effect of the spin reduction from the chiral splitting $\left(\left\langle L_{z}\right\rangle_{d-\bar{d}}=-0.027\right)$. Hence the so called 'cancellation' here is quite different from the total angular momentum case. This difference is due to the orbital contribution in the magnetic moment (25) is $\left\langle L_{z}\right\rangle_{q-\bar{q}}$, but in the nucleon spin sum rule (10d) that is $\left\langle L_{z}\right\rangle_{q+\bar{q}}$.

\section{(b) Decuplet baryons}

Similar to the octet baryons, the decuplet magnetic moments are calculated and the numerical results are listed in Table XI. It should be noted that we use the same set of $\mu_{u}$, $\mu_{d}$ and $\mu_{s}$ as in the octet sector, i.e. no new parameters are introduced.

It is easy to verify from the analytic results given in Table IX that the following equal spacing rules hold for the decuplet magnetic moments,

$$
\begin{aligned}
& \mu_{\Delta^{++}}-\mu_{\Delta^{+}}=\mu_{\Delta^{+}}-\mu_{\Delta^{0}}=\mu_{\Delta^{0}}-\mu_{\Delta^{-}}=\mu_{\Sigma^{*+}}-\mu_{\Sigma^{* 0}}=\mu_{\Sigma^{* 0}}-\mu_{\Sigma^{*-}}=\mu_{\Xi^{* 0}}-\mu_{\Xi^{*-}}, \\
& \mu_{\Delta^{+}}, \mu_{\Sigma^{*+}}=\mu_{\Delta^{0}}-\mu_{\Sigma^{* 0}}=\mu_{\Delta^{-}}-\mu_{\Sigma^{*-}}=\mu_{\Sigma^{* 0}}-\mu_{\Xi^{* 0}}=\mu_{\Sigma^{*-}}-\mu_{\Xi^{*-}}=\mu_{\Xi^{*-}}-\mu_{\Omega^{*-}} .
\end{aligned}
$$


The spacing in Eqs.(29a) and (29b) are 2.82 n.m. and -0.36 n.m. respectively. Our results also show that the decuplet magnetic moments with or without orbital contributions are approximately the same provided the quark magnetic moments change accordingly.

\section{Discussions and Summary}

For the quark flavor and spin observables of the nucleon, the predictions of the chiral quark model are in good agreement with the existing data (see Table IV). We now consider different scenarios of the spin sum rule with or without the gluon contribution.

- Assuming $\left\langle J_{z}>_{G} \simeq 0\right.$, our result shows $\sum_{q}\left\langle L_{z}>_{q+\bar{q}}^{p} \simeq 0.30\right.$, i.e. $60 \%$ of the proton spin is contributed by the orbital motion of quarks and antiquarks. The nucleon spin sum rule (1) becomes

$$
\frac{1}{2}=\sum_{q}<s_{z}>_{q+\bar{q}}(\sim 0.2)+\sum_{q}<L_{z}>_{q+\bar{q}}^{p}(\sim 0.3)+<J_{z}>_{G} \quad(\sim 0) .
$$

- However, if $\left\langle J_{z}\right\rangle_{G}$ is nonzero at low $Q^{2}$ scale as suggested in [41], results given in sections III and IV should be modified. Using the nucleon wave function given in the gluon mixing model [38] and considering the chiral splitting mechanism, we have shown [37] that the ratio of $\sum_{q}\left\langle s_{z}>_{q+\bar{q}}^{p}\right.$ and $\sum_{q}\left\langle L_{z}>_{q+\bar{q}}^{p}\right.$ is the same as that given in (11b). For the parameter set given in (12), this ratio $1 /\left(2 \xi_{1} a\right)-1$ is approximately equal to $2 / 3$. Assuming $<J_{z}>_{G}\left(1 G^{2} V^{2}\right) \simeq 0.15$, we have

$$
\frac{1}{2}=\sum_{q}<s_{z}>_{q+\bar{q}}(\sim 0.14)+\sum_{q}<L_{z}>_{q+\bar{q}}(\sim 0.21)+<J_{z}>_{G}(\sim 0.15) .
$$

- The quantities calculated in the chiral quark model should be viewed as the physical observables at the $Q^{2}$ range lower than $1 \mathrm{GeV}^{2}$. On the other hand, at high $Q^{2}(\geq 1-2$ $\mathrm{GeV}^{2}$ ), the QCD parton model works and the main degrees of freedom are quarks and gluons. According to [14], in the large $Q^{2}$ limit, the partition of the nucleon spin between quarks and gluons follows the well-known partition of the nucleon momentum. It implies that $\sum_{q}<J_{z}>_{q+\bar{q}} \simeq<J_{z}>_{G} \simeq 0.25$, i.e. the nucleon spin is approximately equally shared by quarks (antiquarks) and gluons in the large $Q^{2}$ limit. An interesting question is whether this partition rule holds at the low $Q^{2}$ scale ? We do not know the answer theoretically because it is difficult to solve QCD at this scale. Assuming the partition rule holds at the low $Q^{2}$, then we have third scenario

$$
\frac{1}{2}=\sum_{q}<s_{z}>_{q+\bar{q}}(\sim 0.10)+\sum_{q}<L_{z}>_{q+\bar{q}}(\sim 0.15)+<J_{z}>_{G}(\sim 0.25),
$$

Perhaps experiments in the transition area $Q^{2} \simeq 1-2 \mathrm{GeV}^{2}$ will tell us which scenario is closer to the reality.

In this paper, we discussed the quark flavor, spin and orbital contents in the baryon in the chiral quark model. Contrary to the reduction effect on the quark spin component, the chiral splitting mechanism produces a positive orbital angular momentum shared by quarks 
and antiquarks. Analytical and numerical results for the spin and orbital contents carried by different quark flavors in the baryon are presented. Attention has been drawn to the cancellation between the spin and orbital contributions in the nucleon spin sum rule and in the baryon magnetic moments.

In summary, the chiral quark model with only a few parameters can well explain many nucleon properties: (1) strong flavor asymmetry of light antiquark sea: $\bar{d}>\bar{u}$, (2) nonzero strange quark content, $\left\langle\bar{s} s>\neq 0\right.$, (3) sum of quark spins is small, $\sum_{q}<s_{z}>_{q+\bar{q}} \simeq 0.1-0.2$, (4) sea antiquarks are not polarized: $\Delta \bar{q} \simeq 0(q=u, d, \ldots),(5)$ polarizations of the sea quarks are nonzero and negative, $\Delta q_{\text {sea }}<0$, and (6) the orbital angular momentum of the sea quark is parallel to the proton spin. (1)-(4) are consistent with data (see Table IV), and (5)-(6) could be tested by experiments in the near future.

\section{Acknowledgments}

I would like to thank Xiangdong Ji, and P. K. Kabir for useful comments and suggestions. This work was supported in part by the U. S. Department of Energy and the Institute of Nuclear and Particle Physics, Department of Physics, University of Virginia. 


\section{REFERENCES}

[1] J. Ashman, et al., Nucl. Phys. B328, 1 (1989).

[2] V. W. Hughes and R. Voss, Ann. Rev. Nucl. Part. Sci. 49, 303 (1999).

[3] B. Lampe and E. Reya, Phys. Rep. 332: 1 (2000).

[4] X. Ji, Phys. Rev. Lett. 78, 610 (1997).

[5] P. L. Anthony, et al. Phys. Rev. Lett. 71, 959 (1993);

B. Adeva, et al. Phys. Lett. B302, 533 (1993);

D. Adams, et al., Phys. Lett. B329, 399 (1994);

K. Abe, et al., Phys. Rev. Lett. 74, 346 (1995); 75, 25 (1995); 79, 26 (1997);

K. Ackerstaff, et al., Phys. Lett. B404, 383 (1997).

[6] D. L. Adams, et al., Phys. Lett. B336, 269 (1991).

[7] P. Adams, et al., Phys. Rev. D56, 5330 (1997).

[8] COMPASS proposal, CERN/SPSLC-96-14 (March, 1996);

RHIC Spin Physics Program, (1996);

SLAC-PROPOSAL-E156, (March, 1997).

[9] X. Ji, Phys. Rev. Lett. bf 78, 610 (1997);

X. Ji, W. Melnitchouk, and X. Song, Phys. Rev. D56, 5511 (1997).

[10] M. Gell-Mann, Phys. Lett. 8, 214 (1964).

[11] R. Feynman, Phys. Rev. Lett. 23, 1415 (1969).

[12] J. Babcock, E. Monsay, and D. Sivers, Phys. Rev. 19, 1483 (1979).

[13] P. G. Ratcliffe, Phys. Lett. B192, 180 (1987);

X. Song and J. Du, Phys. Rev. D40, 2177 (1989);

J. W. Qiu, G. P. Ramsey, D. Richards, and D. Sivers, Phys. Rev. D41, 65 (1990).

[14] X. Ji, J. Tang and P. Hoodbhoy, Phys. Rev. Lett. 76, 740 (1996).

[15] S. R. Sharpe, Progress in Lattice Gauge Theory, hep-lat/9811006,

Proc. of Lattice 98, hep-lat/9811023

[16] R. Jaffe and X. Ji, Phys. Rev. D43, 724 (1991).

[17] X. Song and J. S. McCarthy, Phys. Rev. D49, 3169 (1994).

[18] S. Brodsky, J. Ellis and M. Karliner, Phys. Lett. B206, 309 (1988).

[19] L. M. Sehgal, Phys. Rev. D10, 1663 (1974).

[20] M. Casu and L. M. Sehgal, Phys. Rev. D55, 2644 (1997).

[21] E. J. Eichten, I. Hinchliffe and C. Quigg, Phys. Rev. D45, 2269 (1992); Phys. Rev. D47, 747 (1993).

[22] T. P. Cheng and L.-F. Li, Phys. Rev. Lett. 74, 2872 (1995).

[23] X. Song, J. S. McCarthy, and H. J. Weber, Phys. Rev. D55, 2624 (1997).

[24] X. Song, Preprint INPP-UVA-96-05, October, 1996.

[25] H. J. Weber, X. Song, and M. Kirchbach, Mod. Phys. Lett. A12, 729 (1997).

[26] T. P. Cheng and L.-F. Li, Phys. Rev. D57, 344 (1998).

[27] X. Song, Phys. Rev. D57, 4114 (1998).

[28] J. Linde, T. Ohlsson, and H. Snellman, Phys. Rev. D57, 452 (1998); Phys. Rev. D57, 5916 (1998).

[29] T. P. Cheng and L.-F. Li, Phys. Rev. Lett. 80, 2789 (1998).

[30] X. Song, hep-ph/9801332 (INPP-UVA-97-07); hep-ph/9802206 (INPP-UVA-97-08).

[31] M. Gell-Mann, R. J. Oakes, and B. Renner, Phys. Rev. 175, 2194 (1968).

[32] M. Shifman, A. Vainshtein and V. Zakharov, Nucl. Phys. B147, 385, 448 (1979). 
[33] A. E. Dorokhov and N. I. Kochelev, Mod. Phys. Lett. A5, 55 (1990).

[34] Particle Data Group, D. E. Groom, et al., Eur. Phys. J. C15, 1 (2000).

[35] P. Amaudruz et al., Phys. Rev. Lett. 66, 2712 (1991);

M. Arneodo et al., Phys. Rev. D50, R1, (1994).

[36] R. L. Jaffe, Phys. Rev. D54, R6581 (1996);

M. Burkardt and R. L. Jaffe, Phys. Rev. Lett. 70, 2537 (1993).

[37] X. Song, hep-ph/9804461, (INPP-UVA-98-03).

[38] H. J. Lipkin, Phys. Lett. B251, 613 (1990).

[39] H. J. Weber, and K. Bodoor, Int. J. Mod. Phys. E6, 693 (1997).

[40] S. J. Brodsky, and I. Schmidt, Phys. Lett. B234, 144 (1990).

[41] I. Balitsky and X. Ji, Phys. Rev. Lett. 79, 1225 (1997).

[42] K. T. Chao, N. Isgur, and G. Karl, Phys. Rev. D23, 155 (1981).

[43] J. Franklin, Phys. Rev. 182, 1607 (1969); Phys. Rev. D20, 1742 (1979).

[44] J. Bartelski and R. Rodenberg, Phys. Rev. D41, 2800 (1990).

[45] G. Karl, Phys. Rev. D45, 247 (1992).

[46] X. Song and V. Gupta, Phys. Rev. D49, 2211 (1994).

[47] A. Bosshard et al., Phys. Rev. D44, 1962 (1991).

[48] N. B. Wallace et al., Phys. Rev. Lett. 74, 3732 (1995).

[49] H. T. Diehl et al., Phys. Rev. Lett. 67, 804 (1991).

[50] A. Baldit et al., NA51 Collaboration, Phys. Lett. B332, 244 (1994).

[51] J. C. Peng, et al., E866/NuSea Collaboration, Phys. Rev. D58: 092004 (1998).

[52] A. O. Bazarko, et. al, Z. Phys. C65, 189 (1995).

[53] J. Gasser, H. Leutwyler, and M. E. Saino, Phys. Lett. B253, 252 (1991).

[54] S. J. Dong, J.-F. Lagae, and K. F. Liu, Phys. Rev. Lett. 75, 2096 (1995).

[55] B. Adeva et al. Phys. Rev. Lett. B369, 93 (1996). 


\section{TABLES}

TABLE I. The probabilities $P_{q_{\uparrow}}\left(q_{\uparrow, \downarrow}^{\prime}, \bar{q}_{\uparrow, \downarrow}^{\prime}\right)$ and $P_{q_{\uparrow}}\left(q_{\uparrow, \downarrow}^{\prime}, \bar{q}_{\uparrow, \downarrow}^{\prime}\right)$

\begin{tabular}{cccc}
\hline \hline$q^{\prime}$ & $P_{u_{\uparrow}}\left(q_{\uparrow, \downarrow}^{\prime}\right)$ & $P_{d_{\uparrow}}\left(q_{\uparrow, \downarrow}^{\prime}\right)$ & $P_{s_{\uparrow}}\left(q_{\uparrow, \downarrow}^{\prime}\right)$ \\
\hline$u_{\uparrow}$ & $1-\left(\frac{1+\epsilon}{2}+f\right) a+\frac{a}{18}(3-A)^{2}$ & $\frac{a}{18} A^{2}$ & $\frac{a}{18} B^{2}$ \\
$u_{\downarrow}$ & $\left(\frac{1+\epsilon}{2}+f\right) a+\frac{a}{18}(3-A)^{2}$ & $a+\frac{a}{18} A^{2}$ & $\epsilon a+\frac{a}{18} B^{2}$ \\
$d_{\uparrow}$ & $\frac{a}{18} A^{2}$ & $1-\left(\frac{1+\epsilon}{2}+f\right) a+\frac{a}{18}(3-A)^{2}$ & $\frac{a}{18} B^{2}$ \\
$d_{\downarrow}$ & $a+\frac{a}{18} A^{2}$ & $\left(\frac{1+\epsilon}{2}+f\right) a+\frac{a}{18}(3-A)^{2}$ & $\epsilon a+\frac{a}{18} B^{2}$ \\
$s_{\uparrow}$ & $\frac{a}{18} B^{2}$ & $\frac{a}{18} B^{2}$ & $1-\left(\epsilon+f_{s}\right) a+\frac{a}{18} C^{2}$ \\
$s_{\downarrow}$ & $\epsilon a+\frac{a}{18} B^{2}$ & $\epsilon a+\frac{a}{18} B^{2}$ & $\left(\epsilon+f_{s}\right) a+\frac{a}{18} C^{2}$ \\
\hline $\bar{u}_{\uparrow, \downarrow}$ & $\frac{a}{18}(3-A)^{2}$ & $\frac{a}{2}+\frac{a}{18} A^{2}$ & $\frac{\epsilon a}{2}+\frac{a}{18} B^{2}$ \\
$\bar{d}_{\uparrow, \downarrow}$ & $\frac{a}{2}+\frac{a}{18} A^{2}$ & $\frac{a}{18}(3-A)^{2}$ & $\frac{\epsilon a}{2}+\frac{a}{18} B^{2}$ \\
$\bar{s}_{\uparrow, \downarrow}$ & $\frac{\epsilon a}{2}+\frac{a}{18} B^{2}$ & $\frac{\epsilon a}{2}+\frac{a}{18} B^{2}$ & $\frac{a}{18} C^{2}$ \\
\hline \hline
\end{tabular}

TABLE II. The spin-up, spin-down quark (antiquark), spin-average and spin-weighted quark (antiquark) content in the proton. Where $U_{1} \equiv \frac{1}{3}\left[A^{2}+2(3-A)^{2}\right], D_{1} \equiv \frac{1}{3}\left[2 A^{2}+(3-A)^{2}\right]$, and $U_{2}=4 D_{2} \equiv 4(\epsilon+2 f-1)$.

\begin{tabular}{ccc}
\hline \hline \hline$u_{\uparrow}=\frac{5}{3}+\frac{a}{3}\left(-2+\frac{U_{1}}{2}-\frac{U_{2}}{2}\right)$ & $d_{\uparrow}=\frac{1}{3}+\frac{a}{3}\left(2+\frac{D_{1}}{2}+\frac{D_{2}}{2}\right)$ & $s_{\uparrow}=\epsilon a+\frac{a}{3}\left(\frac{B^{2}}{2}\right)$ \\
$u_{\downarrow}=\frac{1}{3}+\frac{a}{3}\left(5+\frac{U_{1}}{2}+\frac{U_{2}}{2}\right)$ & $d_{\downarrow}=\frac{2}{3}+\frac{a}{3}\left(4+\frac{D_{1}}{2}-\frac{D_{2}}{2}\right)$ & $s_{\downarrow}=2 \epsilon a+\frac{a}{3}\left(\frac{B^{2}}{2}\right)$ \\
\hline $\bar{u}_{\uparrow}=\bar{u}_{\downarrow}=\frac{a}{2}+\frac{a}{3}\left(\frac{U_{1}}{2}\right)$ & $\bar{d}_{\uparrow}=\bar{d}_{\downarrow}=a+\frac{a}{3}\left(\frac{D_{1}}{2}\right)$ & $\bar{s}_{\uparrow}=\bar{s}_{\downarrow}=\frac{3 \epsilon a}{2}+\frac{a}{3}\left(\frac{B^{2}}{2}\right)$ \\
\hline \hline$u=2+\frac{a}{3}\left(3+U_{1}\right)$ & $d=1+\frac{a}{3}\left(6+D_{1}\right)$ & $s=3 \epsilon a+\frac{a}{3} B^{2}$ \\
\hline $\bar{u}=\frac{a}{3}\left(3+U_{1}\right)$ & $\bar{d}=\frac{a}{3}\left(6+D_{1}\right)$ & $\bar{s}=3 \epsilon a+\frac{a}{3} B^{2}$ \\
\hline \hline$\Delta u=\frac{4}{3}[1-a(\epsilon+2 f)]-a$ & $\Delta d=\frac{-1}{3}[1-a(\epsilon+2 f)]-a$ & $\Delta s=a(1-\epsilon)-a$ \\
\hline$\Delta \bar{u}=0$ & $\Delta \bar{d}=0$ & $\Delta \bar{s}=0$ \\
\hline \hline
\end{tabular}

TABLE III. The orbital angular momentum carried by the quark $q^{\prime}\left(\bar{q}^{\prime}\right)$, spin-up and -down are included, from a valence spin-up quark $q_{\uparrow}$ fluctuates into all allowed final states. Where $\delta \equiv(1-3 \kappa) / \kappa$.

\begin{tabular}{lccc}
\hline \hline & $<L_{z}>_{q^{\prime}, \bar{q}^{\prime} / u_{\uparrow}}$ & $<L_{z}>_{q^{\prime}, \bar{q}^{\prime} / d_{\uparrow}}$ & $<L_{z}>_{q^{\prime}, \bar{q}^{\prime} / s_{\uparrow}}$ \\
\hline$q^{\prime}=u$ & $\kappa a\left[\xi_{1}+f \delta+\frac{(3-A)^{2}}{9}\right]$ & $\kappa a\left[1+\delta+\frac{A^{2}}{9}\right]$ & $\kappa a\left[\epsilon(1+\delta)+\frac{B^{2}}{9}\right]$ \\
$q^{\prime}=d$ & $\kappa a\left[1+\delta+\frac{A^{2}}{9}\right]$ & $\kappa a\left[\xi_{1}+f \delta+\frac{(3-A)^{2}}{9}\right]$ & $\kappa a\left[\epsilon(1+\delta)+\frac{B^{2}}{9}\right]$ \\
$q^{\prime}=s$ & $\kappa a\left[\epsilon(1+\delta)+\frac{B^{2}}{9}\right]$ & $\kappa a\left[\epsilon(1+\delta)+\frac{B^{2}}{9}\right]$ & $\kappa a\left[\xi_{2}+f_{s} \delta+\frac{C^{2}}{9}\right]$ \\
\hline $\bar{q}^{\prime}=\bar{u}$ & $\kappa a\left[\frac{(3-A)^{2}}{9}\right]$ & $\kappa a\left[1+\frac{A^{2}}{9}\right]$ & $\kappa a\left[\epsilon+\frac{B^{2}}{9}\right]$ \\
$\bar{q}^{\prime}=\bar{d}$ & $\kappa a\left[1+\frac{A^{2}}{9}\right]$ & $\kappa a\left[\frac{(3-A)^{2}}{9}\right]$ & $\kappa a\left[\epsilon+\frac{B^{2}}{9}\right]$ \\
$\bar{q}^{\prime}=\bar{s}$ & $\kappa a\left[\epsilon+\frac{B^{2}}{9}\right]$ & $\kappa a\left[\epsilon+\frac{B^{2}}{9}\right]$ & $\kappa a\left[\frac{C^{2}}{9}\right]$ \\
\hline \hline
\end{tabular}


TABLE IV. Quark spin and flavor observables in the proton. The quantity used as input is indicated by a star.

\begin{tabular}{|c|c|c|c|}
\hline Quantity & Data & This paper & NQM \\
\hline \multirow[t]{2}{*}{$\bar{d}-\bar{u}$} & $0.147 \pm 0.039$ & $0.143^{*}$ & 0 \\
\hline & $0.100 \pm 0.018$ & & \\
\hline $\bar{u} / \bar{d}$ & $\begin{array}{c}{\left[\frac{\bar{u}(x)}{d(x)}\right]_{x=0.18}=0.51 \pm 0.06} \\
{\left[\frac{\bar{u}(x)}{\bar{d}(x)}\right]_{0.1<x<0.2}=0.67 \pm 0.06}\end{array}$ & 0.64 & - \\
\hline $2 \bar{s} /(\bar{u}+\bar{d})$ & $\frac{<2 x \bar{s}(x)>}{<x(\bar{u}(x)+d(x))>}=0.477 \pm 0.051$ 52 & 0.72 & - \\
\hline $2 \bar{s} /(u+d)$ & $\frac{<2 x \bar{s}(x)>}{<x(u(x)+d(x))>}=0.099 \pm 0.009$ & 0.13 & 0 \\
\hline$\sum \bar{q} / \sum q$ & $\frac{\sum<x \bar{q}(x)>}{\sum<x q(x)>}=0.245 \pm 0.0055$ & 0.23 & 0 \\
\hline \multirow[t]{3}{*}{$f_{s}$} & $0.10 \pm 0.06[53]$ & 0.10 & 0 \\
\hline & $0.15 \pm 0.03$ & & \\
\hline & $\frac{<2 x \bar{s}(x)>}{\sum<x(q(x)+\bar{q}(x))>}=0.076 \pm 0.022$ 52 & & \\
\hline$f_{3} / f_{8}$ & $0.21 \pm 0.05[22$ & 0.22 & $1 / 3$ \\
\hline$\Delta u$ & $0.85 \pm 0.05$ ]7] & 0.86 & $4 / 3$ \\
\hline$\Delta d$ & $-0.41 \pm 0.05[\overline{6]}]$ & -0.40 & $-1 / 3$ \\
\hline$\Delta s$ & $-0.07 \pm 0.05[\overline{7]}$ & -0.07 & 0 \\
\hline$\Delta \bar{u}, \Delta \bar{d}$ & $-0.02 \pm 0.115$ & 0 & 0 \\
\hline$\Delta_{3} / \Delta_{8}$ & $2.17 \pm 0.10$ & 2.12 & $5 / 3$ \\
\hline$\Delta_{3}$ & $1.2601 \pm 0.0028$ & $1.26^{*}$ & $5 / 3$ \\
\hline$\Delta_{8}$ & $0.579 \pm 0.025$ & $0.60^{*}$ & 1 \\
\hline
\end{tabular}

TABLE V. Quark spin and orbital angular momentum in the proton in different models.

\begin{tabular}{ccccccc}
\hline \hline Quantity & Data [0] & \multicolumn{3}{c}{ This paper } & Sehgal [19] & NQM \\
& & $\kappa=1 / 4$ & $\kappa=1 / 3$ & $\kappa=3 / 8$ & & \\
\hline$<L_{z}>_{u}^{p}$ & - & 0.115 & 0.130 & 0.138 & - & 0 \\
$<L_{z}>_{d}^{p}$ & - & 0.073 & 0.043 & 0.027 & - & 0 \\
$<L_{z}>_{s}^{p}$ & - & 0.038 & 0.028 & 0.023 & - & 0 \\
$<L_{z}>_{\bar{p}}^{p}$ & - & -0.003 & -0.003 & -0.004 & - & 0 \\
$<L_{z}>_{\bar{p}}^{p}$ & - & 0.057 & 0.076 & 0.086 & - & 0 \\
$<L_{z}>_{\bar{p}}^{p}$ & - & 0.021 & 0.028 & 0.031 & - & 0 \\
\hline$\sum_{q}<L_{z}>_{q+\bar{q}}^{p}$ & - & 0.30 & 0.30 & 0.30 & 0.39 & 0 \\
\hline$\Delta u^{p}$ & $0.85 \pm 0.05$ & & 0.86 & & 0.78 & $4 / 3$ \\
$\Delta d^{p}$ & $-0.41 \pm 0.05$ & $-0.07 \pm 0.05$ & -0.40 & & -0.34 & $-1 / 3$ \\
$\Delta s^{p}$ & $0.19 \pm 0.06$ & -0.07 & & -0.14 & 0 \\
\hline$\frac{1}{2} \Delta \Sigma^{p}$ &
\end{tabular}


TABLE VI. Quark spin and orbital angular momentum in different models.

\begin{tabular}{lccccc}
\hline \hline & NQM & MIT bag & This paper & CS [20] & Skyrme \\
\hline$\sum_{q}<s_{z}>_{q+\bar{q}}^{p}$ & $1 / 2$ & 0.32 & 0.20 & 0.08 & 0 \\
\hline$\sum_{q}<L_{z}>_{q+\bar{q}}^{p}$ & 0 & 0.18 & 0.30 & 0.42 & $1 / 2$ \\
\hline \hline
\end{tabular}

TABLE VII. The quark spin and orbital content in the octet baryons. Where $\xi_{1}=1+\epsilon+f$, $\xi_{2}=2 \epsilon+f_{s}$, and $\delta \equiv(1-3 \kappa) / \kappa$.

\begin{tabular}{cccc}
\hline \hline Baryon & $\Delta u^{B}$ & $\Delta d^{B}$ & $\Delta s^{B}$ \\
\hline $\mathrm{p}$ & $\frac{4}{3}-\frac{a}{3}\left(8 \xi_{1}-4 \epsilon-5\right)$ & $-\frac{1}{3}-\frac{a}{3}\left(-2 \xi_{1}+\epsilon+5\right)$ & $-a \epsilon$ \\
$\Sigma^{+}$ & $\frac{4}{3}-\frac{a}{3}\left(8 \xi_{1}-5 \epsilon-4\right)$ & $-\frac{a}{3}(4-\epsilon)$ & $-\frac{1}{3}-\frac{2 a}{3}\left(3 \epsilon-\xi_{2}\right)$ \\
$\Sigma^{0}$ & $\frac{2}{3}-\frac{a}{3}\left(4 \xi_{1}-3 \epsilon\right)$ & $\frac{2}{3}-\frac{a}{3}\left(4 \xi_{1}-3 \epsilon\right)$ & $-\frac{1}{3}-\frac{2 a}{3}\left(3 \epsilon-\xi_{2}\right)$ \\
$\Lambda^{0}$ & $-a \epsilon$ & $-a \epsilon$ & $1-2 a\left(\xi_{2}-\epsilon\right)$ \\
$\Xi^{0}$ & $-\frac{1}{3}-\frac{a}{3}\left(-2 \xi_{1}+5 \epsilon+1\right)$ & $-\frac{a}{3}(4 \epsilon-1)$ & $\frac{4}{3}-\frac{a}{3}\left(8 \xi_{2}-9 \epsilon\right)$ \\
\hline & $\sum_{q}<L_{z}>_{q}^{B}$ & $\sum_{\bar{q}}<L_{z}>\bar{B}$ & $\sum_{q}<L_{z}>{ }_{q+\bar{q}}^{B}$ \\
\hline $\mathrm{p}$ & $(2+\delta) \kappa a \xi_{1}$ & $\kappa a \xi_{1}$ & $a \xi_{1}$ \\
$\Sigma^{+}$ & $(2+\delta) \frac{\kappa a}{3}\left(4 \xi_{1}-\xi_{2}\right)$ & $\frac{\kappa a}{3}\left(4 \xi_{1}-\xi_{2}\right)$ & $\frac{a}{3}\left(4 \xi_{1}-\xi_{2}\right)$ \\
$\Lambda^{0}$ & $(2+\delta) \kappa a \xi_{2}$ & $\kappa a \xi_{2}$ & $a \xi_{2}$ \\
$\Xi^{0}$ & $(2+\delta) \frac{\kappa a}{3}\left(4 \xi_{2}-\xi_{1}\right)$ & $\frac{\kappa a}{3}\left(4 \xi_{2}-\xi_{1}\right)$ & $\frac{a}{3}\left(4 \xi_{2}-\xi_{1}\right)$ \\
\hline \hline
\end{tabular}

TABLE VIII. Quark spin and orbital angular momentum in other octet baryons.

\begin{tabular}{|c|c|c|c|c|c|c|c|c|c|}
\hline \multirow[t]{2}{*}{ Baryon } & \multicolumn{3}{|c|}{$\Sigma^{+}$} & \multicolumn{3}{|c|}{$\Lambda$} & \multicolumn{3}{|c|}{$\Xi^{0}$} \\
\hline & $\kappa=1 / 4$ & $\kappa=1 / 3$ & $\kappa=3 / 8$ & $\kappa=1 / 4$ & $\kappa=1 / 3$ & $\kappa=3 / 8$ & $\kappa=1 / 4$ & $\kappa=1 / 3$ & $\kappa=3 / 8$ \\
\hline$<L_{z}>_{u}^{B}$ & 0.130 & 0.141 & 0.147 & 0.038 & 0.028 & 0.023 & 0.014 & 0.000 & -0.008 \\
\hline$<L_{z}>_{d}^{B}$ & 0.096 & 0.071 & 0.058 & 0.038 & 0.028 & 0.023 & 0.023 & 0.017 & 0.014 \\
\hline$<L_{z}>_{s}^{B}$ & 0.029 & 0.015 & 0.007 & 0.063 & 0.067 & 0.069 & 0.071 & 0.080 & 0.085 \\
\hline$<L_{z}>\frac{B}{\bar{u}}$ & 0.005 & 0.007 & 0.008 & 0.021 & 0.028 & 0.031 & 0.025 & 0.033 & 0.037 \\
\hline$<L_{z}>\frac{B}{\bar{d}}$ & 0.053 & 0.071 & 0.079 & 0.021 & 0.028 & 0.031 & 0.013 & 0.017 & 0.019 \\
\hline$<L_{z}>_{\bar{s}}^{\frac{B}{S}}$ & 0.026 & 0.035 & 0.039 & 0.004 & 0.006 & 0.007 & -0.001 & -0.001 & -0.002 \\
\hline$\sum_{q}<L_{z}>_{q+\bar{q}}^{B}$ & 0.34 & 0.34 & 0.34 & 0.18 & 0.18 & 0.18 & 0.15 & 0.15 & 0.15 \\
\hline$\Delta u^{B}$ & & 0.84 & & & -0.07 & & & -0.29 & \\
\hline$\Delta d^{B}$ & & -0.17 & & & -0.07 & & & -0.04 & \\
\hline$\Delta s^{B}$ & & -0.35 & & & 0.77 & & & 1.05 & \\
\hline$\frac{1}{2} \Delta \Sigma^{B}$ & & 0.16 & & & 0.32 & & & 0.35 & \\
\hline
\end{tabular}


TABLE IX. The quark spin and orbital content in the decuplet baryons.

\begin{tabular}{cccc}
\hline \hline Baryon & $\Delta u^{B^{*}}$ & $\Delta d^{B^{*}}$ & $\Delta s^{B^{*}}$ \\
\hline$\Delta^{++}$ & $3-3 a\left(2 \xi_{1}-\epsilon-1\right)$ & $-3 a$ & $-3 a \epsilon$ \\
$\Delta^{+}$ & $2-a\left(4 \xi_{1}-2 \epsilon-1\right)$ & $1-a\left(2 \xi_{1}-\epsilon+1\right)$ & $-3 a \epsilon$ \\
$\Sigma^{* 0}$ & $1-2 a \xi_{1}$ & $1-2 a \xi_{1}$ & $1-2 a \xi_{2}$ \\
$\Sigma^{*+}$ & $2-a\left(4 \xi_{1}-\epsilon-2\right)$ & $-a(\epsilon+2)$ & $1-2 a \xi_{2}$ \\
$\Xi^{* 0}$ & $1-a\left(2 \xi_{1}+\epsilon-1\right)$ & $-a(2 \epsilon+1)$ & $2-a\left(4 \xi_{2}-3 \epsilon\right)$ \\
$\Omega^{-}$ & $-3 a \epsilon$ & $-3 a \epsilon$ & $3-6 a\left(\xi_{2}-\epsilon\right)$ \\
\hline & $\sum_{q}<L_{z}>_{q}^{B^{*}}$ & $\sum_{\bar{q}}<L_{z}>_{\bar{q}}^{B^{*}}$ & $\sum_{q}<L_{z}>_{q+\bar{q}}^{B^{*}}$ \\
\hline$\Delta$ & $(2+\delta) \kappa a\left(3 \xi_{1}\right)$ & $\kappa a\left(3 \xi_{1}\right)$ & $a\left(3 \xi_{1}\right)$ \\
$\Sigma$ & $(2+\delta) \kappa a\left(2 \xi_{1}+\xi_{2}\right)$ & $\kappa a\left(2 \xi_{1}+\xi_{2}\right)$ & $a\left(2 \xi_{1}+\xi_{2}\right)$ \\
$\Xi$ & $(2+\delta) \kappa a\left(\xi_{1}+2 \xi_{2}\right)$ & $\kappa a\left(\xi_{1}+2 \xi_{2}\right)$ & $a\left(\xi_{1}+2 \xi_{2}\right)$ \\
$\Omega$ & $(2+\delta) \kappa a\left(3 \xi_{2}\right)$ & $\kappa a\left(3 \xi_{2}\right)$ & $a\left(3 \xi_{2}\right)$ \\
\hline \hline
\end{tabular}

TABLE X. Comparison of our predictions with data for the octet baryon magnetic moments. The naive quark model (NQM) results are also listed. The quantity used as input is indicated by a star.

\begin{tabular}{|c|c|c|c|c|c|}
\hline \multirow[t]{2}{*}{ Baryon } & \multirow[t]{2}{*}{ data } & \multicolumn{3}{|c|}{ This paper } & \multirow[t]{2}{*}{ NQM } \\
\hline & & $\kappa=1 / 4$ & $\kappa=1 / 3$ & $\kappa=3 / 8$ & \\
\hline $\mathrm{p}$ & $2.79 \pm 0.00$ & & $2.79^{*}$ & & $2.79^{*}$ \\
\hline $\mathrm{n}$ & $-1.91 \pm 0.00$ & & $-1.91^{*}$ & & $-1.91^{*}$ \\
\hline$\Sigma^{+}$ & $2.46 \pm 0.01$ & & 2.67 & & 2.67 \\
\hline$\Sigma^{-}$ & $-1.16 \pm 0.03$ & & -1.09 & & -1.09 \\
\hline$\Lambda^{0}$ & $-0.61 \pm 0.00$ & & $-0.61^{*}$ & & $-0.61^{*}$ \\
\hline$\Xi^{0}$ & $-1.25 \pm 0.01$ & & -1.43 & & -1.43 \\
\hline$\Xi^{-}$ & $-0.65 \pm 0.00$ & & -0.49 & & -0.49 \\
\hline$\mu_{u}$ & & 2.404 & 2.351 & 2.328 & 1.850 \\
\hline$\mu_{d}$ & & -1.047 & -0.944 & -0.892 & -0.972 \\
\hline$\mu_{s}$ & & -0.657 & -0.623 & -0.606 & -0.613 \\
\hline
\end{tabular}


TABLE XI. Comparison of our predictions with data for the decuplet baryon magnetic moments. The naive quark model (NQM) results are also listed.

\begin{tabular}{cccc}
\hline \hline Baryon & data & $\begin{array}{c}\text { This paper } \\
\kappa=1 / 3\end{array}$ & NQM \\
\hline$\Delta^{++}$ & $4.52 \pm 0.50 \pm 0.45[47]$ & 5.55 & 5.58 \\
& $3.7<\mu_{\Delta^{++}}<7.5[34]$ & & \\
$\Delta^{+}$ & - & 2.73 & 2.79 \\
$\Delta^{0}$ & - & -0.09 & 0.00 \\
$\Delta^{-}$ & - & -2.91 & -2.79 \\
$\Sigma^{*+}$ & - & 3.09 & 3.11 \\
$\Sigma^{* 0}$ & - & 0.27 & 0.32 \\
$\Sigma^{*-}$ & - & -2.55 & -2.47 \\
$\Xi^{* 0}$ & - & 0.63 & 0.64 \\
$\Xi^{*-}$ & - & -2.19 & -2.15 \\
$\Omega^{-}$ & $-1.94 \pm 0.17 \pm 0.14[49]$ & -1.83 & -1.83 \\
& $-2.024 \pm 0.056[48]$ & & \\
\hline$\mu_{u}$ & $-2.02 \pm 0.05[34]$ & 2.351 & 1.85 \\
$\mu_{d}$ & & -0.944 & -0.97 \\
$\mu_{s}$ & & -0.623 & -0.61 \\
\hline \hline
\end{tabular}




\section{FIGURES}

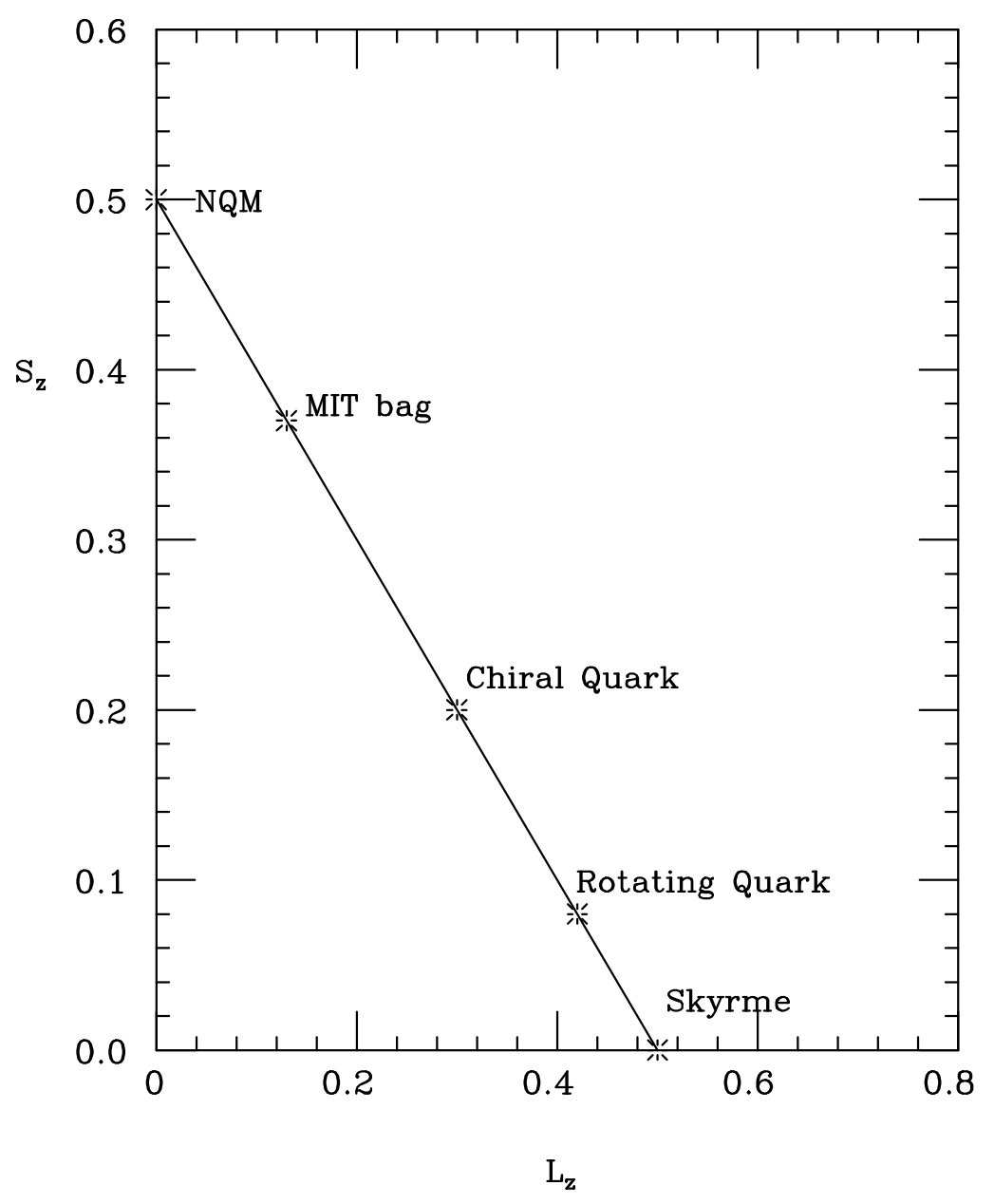

FIG. 1. Quark spin and orbital angular momentum $\left(\sum_{q}<s_{z}>_{q+\bar{q}}\right.$ versus $\left.\sum_{q}<L_{z}>_{q+\bar{q}}\right)$ in the nucleon in different models. 


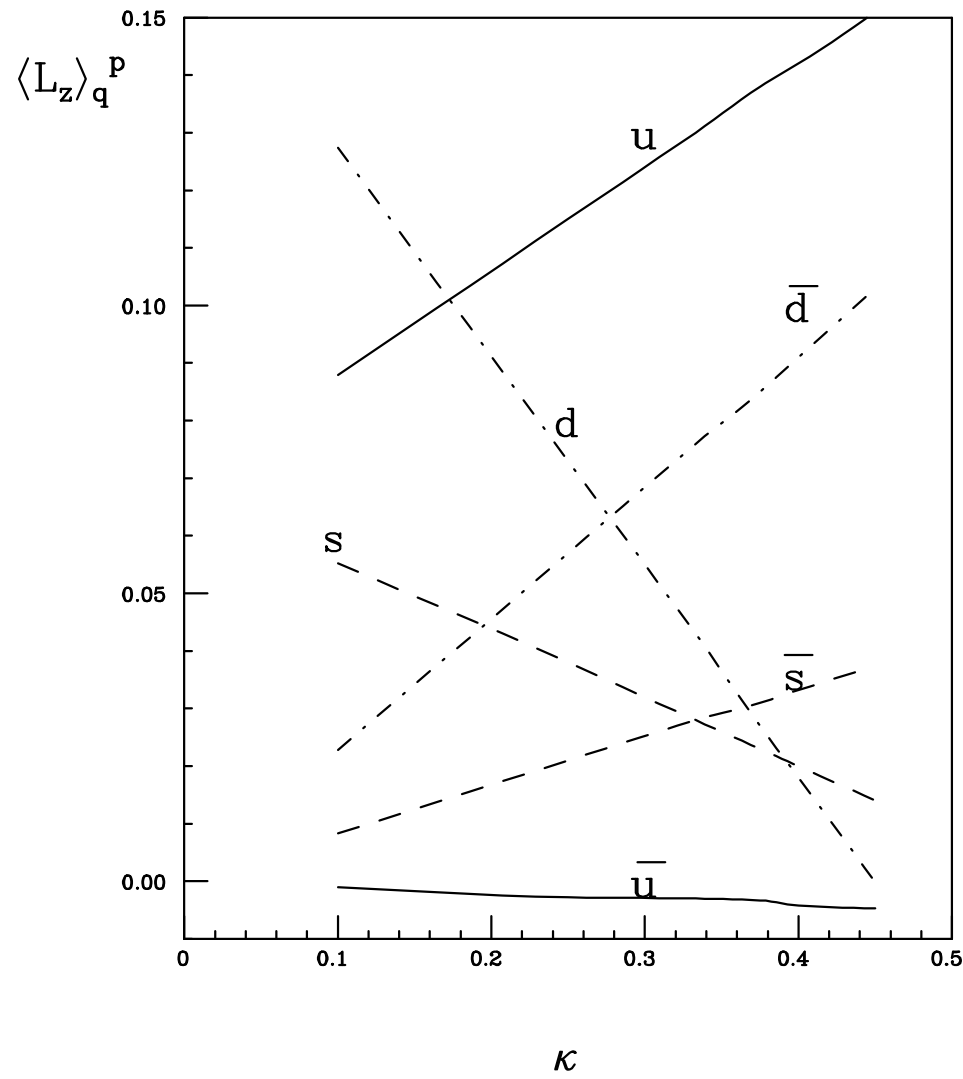

FIG. 2. Quark or antiquark orbital angular momentum $\left\langle L_{z}\right\rangle_{q, \bar{q}}$ in the proton as function of the partition factor $\kappa$. 


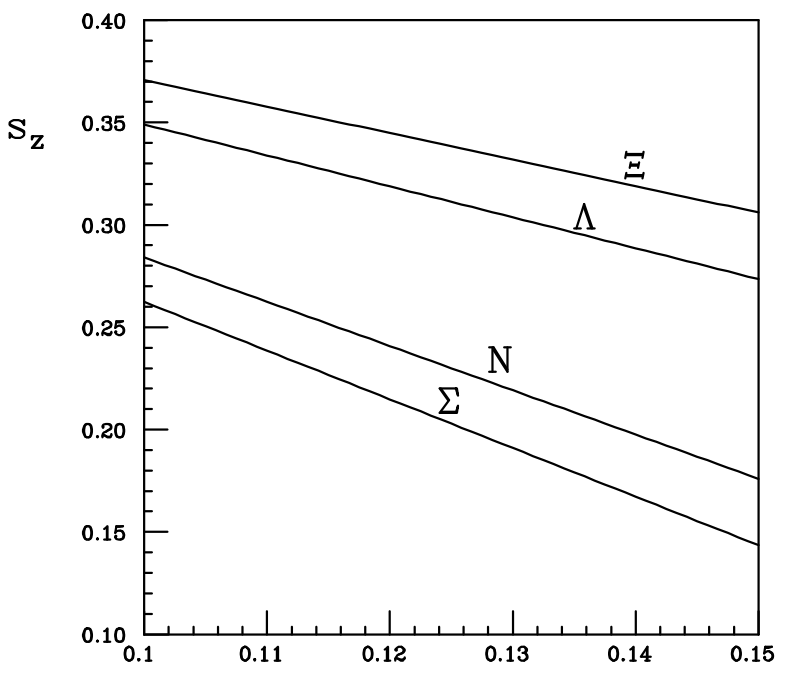

a

FIG. 3. Quark spin content $\left(\sum_{q}<s_{z}>_{q+\bar{q}}^{B}\right)$ in different octet baryons as function of $a$ 